\title{
Seed fields for galactic dynamos by the magnetorotational instability
}

\author{
L. L. Kitchatinov ${ }^{1,2}$ and G. Rüdiger ${ }^{1}$ \\ 1 Astrophysikalisches Institut Potsdam, An der Sternwarte 16, 14482, Potsdam, Germany \\ e-mail: [gruediger;1kitchatinov]@aip.de \\ 2 Institute for Solar-Terrestrial Physics, PO Box 4026, Irkutsk 664033, Russian Federation \\ e-mail: kit@iszf.irk.ru
}

Received 12 March 2004 / Accepted 13 May 2004

\begin{abstract}
A linear but global numerical model for the magnetorotational instability (MRI) in a disk geometry is considered to estimate the instability parameters for galaxies. Similarity rules suggested by a local analysis are applied to reveal a universal behavior of the results of global calculations for large magnetic Prandtl numbers. The findings are used to estimate the MRI characteristics for galaxies with their very large magnetic Prandtl numbers which cannot be attained in any numerical simulations. The resulting minimum field for the instability, $B_{\min } \simeq 10^{-25} \mathrm{G}$, is small compared to any seed fields currently discussed. The growth times of MRI are estimated to be on the order of the rotation period of the inner rigidly-rotating core, i.e. $\sim 100$ Myr. Global MRI excites preferentially magnetic field modes of quadrupolar symmetry.
\end{abstract}

Key words. magnetohydrodynamics (MHD) - galaxies: magnetic fields - galaxies: evolution

\section{Introduction}

The magnetorotational instability (MRI) has been discovered by Velikhov (1959) for hydromagnetic Couette flows; in the astrophysical context it has been established by Fricke (1969) and Balbus \& Hawley (1991). Several properties of the instability make it also relevant for the theory of galactic magnetism.

The instability is due to magnetic fields, but the minimum field for MRI is really very small. We shall see that for galactic conditions the minimum field, $B_{\min } \simeq 10^{-25} \mathrm{G}$, is much smaller than the seed magnetic fields of protogalaxies, whatever origin of the seed fields we assume. With its growth time of order the rotation time, the MRI is fast, with e-folding times below 0.1 Gyr. The observation of Faraday rotation of highredshift objects by Athreya et al. (1998) and Carilli \& Taylor (2002) seems to indicate that such short growth times really exist (Lesch \& Hanasz 2003).

It was recognized by Beck et al. (1994) that the seed field can be largely amplified by interstellar turbulence before the global hydromagnetic dynamo starts operating (Cattaneo 1999; Schekochihin et al. 2004). Turbulent velocities of about $10 \mathrm{~km} \mathrm{~s}^{-1}$ are indeed indicated by spectral line broadening of protogalactic disks (Wolfe 1988). The origin of the turbulence is, however, uncertain. Supernova explosions would drive a much weaker turbulence (Ferrière 1992; Kaisig et al. 1993). Another driver is MRI which was demonstrated by Sellwood $\&$ Balbus (1999) to produce its own turbulence in the nonlinear regime.
A more specific problem of galactic dynamos to which MRI can also be relevant is the parity selection by the global magnetic dynamo. Galactic dynamo models prefer global fields symmetric about the disk midplane (Stix 1975, and many other references in Rüdiger \& Hollerbach 2004). This symmetry type is also favored by observations. In order to be amplified, however, the quadrupolar field should already be present in the seed field for the dynamo. Not all origins for the seed field currently discussed lead to the required symmetry, e.g., the battery effect of Biermann gives rise to dipolar global fields (Krause \& Beck 1998). We shall demonstrate that MRI excites preferentially global modes with quadrupolar parity even if the (axial) destabilizing field is antisymmetric with respect to the disk midplane. This property suggests MRI as a natural way to produce the seed field for global galactic dynamos.

One could argue that MRI in the weak-field regime is rather local by nature and not efficient in producing global patterns. The local theory of ideal fluids yields a growth rate, $\gamma$, for MRI proportional to the Alfvén frequency, $\gamma \sim V_{\mathrm{A}} k$, in the weak-field case, $V_{\mathrm{A}} \ll \Omega / k\left(V_{\mathrm{A}}=B_{0} / \sqrt{\mu_{0} \rho}\right.$ is the Alfvén velocity, $k$ is the vertical wave number, $\Omega$ is the local angular velocity, and $B_{0}$ is the background axial magnetic field). The growth rate seemingly increases with wave number. The growth is, however, stabilized by the microscopic magnetic diffusivity, $\eta$, so that $\gamma+\eta k^{2} \simeq V_{\mathrm{A}} k$. Marginal stability then demands $V_{\mathrm{A}} \simeq \eta k$. The smallest field strength producing the instability belongs to the smallest possible $k$. The low bound on the magnetic field producing MRI is therefore controlled by the global geometry. 
We try to find out in the present paper whether MRI should be expected for protogalaxies where only very weak seed fields are present and the magnetic Prandtl number is extremely large (see Kulsrud \& Anderson 1992). The extent to which the seed field can be amplified by the turbulence is evaluated. The preferred geometry of the magnetic field in the global unstable modes is determined. To this end the disk model of Kitchatinov \& Mazur (1997) is used which is outlined in the next section. Section 3 presents the results of the computations. The implications for galactic dynamos are discussed in Sect. 4.

\section{The model}

The model concerns a rotating disk of constant thickness, $2 \mathrm{H}$, threaded by a uniform axial magnetic field. The rotation axis is normal to the disk and the angular velocity, $\Omega$, depends on the distance, $s$, to the axis. This dependence is parameterized by

$\Omega(s)=\Omega_{0} \tilde{\Omega}(s)$

with

$\tilde{\Omega}(s)=\left(1+\left(\frac{s}{s_{0}}\right)^{n}\right)^{-1 / n}$.

This profile describes almost uniform rotation up to the distance $s_{0}$, and the law $\Omega \simeq \Omega_{0}\left(s_{0} / s\right)$, implying constant rotation velocity for distances $s \gg s_{0}$, as is observed for nearly all galaxies. We use $n=2$ in Eq. (2) and $s_{0} / H=5$ for the aspect ratio.

We assume an incompressible fluid, $\operatorname{div} \boldsymbol{U}=0$. The fluid compressibility is probably not important for our analysis because MRI proceeds via noncompressive Alfvén-type disturbances. The magnetic field and the flow in the disk obey the induction equation,

$\frac{\partial \boldsymbol{B}}{\partial t}=\operatorname{curl}(\boldsymbol{U} \times \boldsymbol{B})+\eta \Delta \boldsymbol{B}$,

and the equation for vorticity, $\boldsymbol{W}=\operatorname{curl} \boldsymbol{U}$,

$$
\frac{\partial \boldsymbol{W}}{\partial t}=\operatorname{curl}(\boldsymbol{U} \times \boldsymbol{W}+\boldsymbol{J} \times \boldsymbol{B} / \rho)+v \Delta \boldsymbol{W}
$$

which results from taking the curl of the momentum equation to eliminate the pressure. $\boldsymbol{J}=\left(1 / \mu_{0}\right)$ curl $\boldsymbol{B}$ is the current density.

To perform the linear stability analysis, we linearize Eqs. (3) and (4) about the rotation (2) and the uniform axial field, $\boldsymbol{B}_{0}=B_{0} \hat{\boldsymbol{e}}_{z}$ ( $\hat{\boldsymbol{e}}_{z}$ is the unit vector along the rotation axis), and introduce the normalized variables,

$\boldsymbol{b}=\boldsymbol{B}^{\prime} / B_{0}, \boldsymbol{j}=\frac{\mu_{0} H}{B_{0}} \boldsymbol{J}^{\prime}, \boldsymbol{u}=\boldsymbol{U}^{\prime} /\left(H \Omega_{0}\right), \boldsymbol{\omega}=\boldsymbol{W}^{\prime} / \Omega_{0}$,

where dimensional disturbances are dashed. This leads to the three basic dimensionless parameters of magnetic Reynolds number, Rm, Hartmann number, Ha, and magnetic Prandtl number, Pm,

$$
\mathrm{Rm}=\frac{\Omega_{0} H^{2}}{\eta}, \quad \mathrm{Ha}=\frac{B_{0} H}{\sqrt{\mu_{0} \rho v \eta}}=\frac{V_{\mathrm{A}} H}{\sqrt{v \eta}}, \quad \mathrm{Pm}=\frac{v}{\eta} .
$$

They control the equation system for the normalized disturbances,

$$
\begin{aligned}
\frac{\partial \boldsymbol{b}}{\partial t}= & \operatorname{Rm} \operatorname{curl}\left(s \tilde{\Omega}(s) \hat{\boldsymbol{e}}_{\phi} \times \boldsymbol{b}-\hat{\boldsymbol{e}}_{z} \times \boldsymbol{u}\right)+\Delta \boldsymbol{b} \\
\frac{\partial \boldsymbol{\omega}}{\partial t}= & \operatorname{Rm} \operatorname{curl}\left(s \tilde{\Omega}(s) \hat{\boldsymbol{e}}_{\phi} \times \omega-\frac{\kappa^{2}}{2 \tilde{\Omega}(s)} \hat{\boldsymbol{e}}_{z} \times \boldsymbol{u}\right) \\
& -\frac{\mathrm{Pm} \mathrm{Ha}^{2}}{\mathrm{Rm}} \operatorname{curl}\left(\hat{\boldsymbol{e}}_{z} \times \boldsymbol{j}\right)+\operatorname{Pm} \Delta \omega
\end{aligned}
$$

where $\kappa$ is the normalized epicycle frequency

$$
\kappa^{2}=\frac{2 \tilde{\Omega}}{s} \frac{\mathrm{d} s^{2} \tilde{\Omega}}{\mathrm{d} s},
$$

time and distances are normalized to the diffusive time, $H^{2} / \eta$, and to the disk half-thickness, $H$, respectively. The boundary conditions on the disk surfaces are (i) stress-free for the flow and (ii) pseudo-vacuum condition for the magnetic disturbances, i.e.

$\hat{\boldsymbol{e}}_{z} \times \boldsymbol{b}=0$.

The solutions are required to be regular at the rotation axis and to vanish at infinity.

The linear stability analysis with $\boldsymbol{b}, \boldsymbol{\omega} \sim \exp (\gamma t)$ leads to an eigenvalue problem for the equation system (7). The problem has been solved numerically with the method developed by Kitchatinov \& Mazur (1997). As usual, the system (7) allows two types of solutions with different symmetries about the disk midplane. One of the symmetry types combines a symmetric magnetic field with an antisymmetric flow field. This symmetry type is called "quadrupolar" (we are mainly interested in the symmetry of the magnetic pattern). The other symmetry type of "dipolar" perturbations combines antisymmetric magnetic fields with symmetric flows. The eigenmodes are also distinguished by their azimuthal wave number, $m$, because the axisymmetric modes with $m=0$ and nonaxisymmetric modes with $m \geq 1$ satisfy independent equation systems.

The primary aim of the linear theory is to define the stability boundary in parameter space which separates the region of stable perturbations with negative or zero real part of $\gamma$ from the instability region with (exponentially) growing perturbations. The stability boundary depends on the symmetry type of the excitations. The growth rates of the unstable disturbances are less relevant, since they characterize states of the system that are unlikely to be present precisely just because they are unstable. The instability region is the realm of nonlinear simulations.

\section{Results}

Because of the galactic implications, we focus on the model results for large magnetic Prandtl numbers, Pm $\gg 1$. Figure 1 shows neutral stability lines for axisymmetric quadrupolar modes. As discussed below, modes of this symmetry type are excited preferentially. The instability region is located between the left and right branches, which define the minimum and maximum field strengths for the instability, respectively. The minima of the lines define the minimum Reynolds number for instability. 


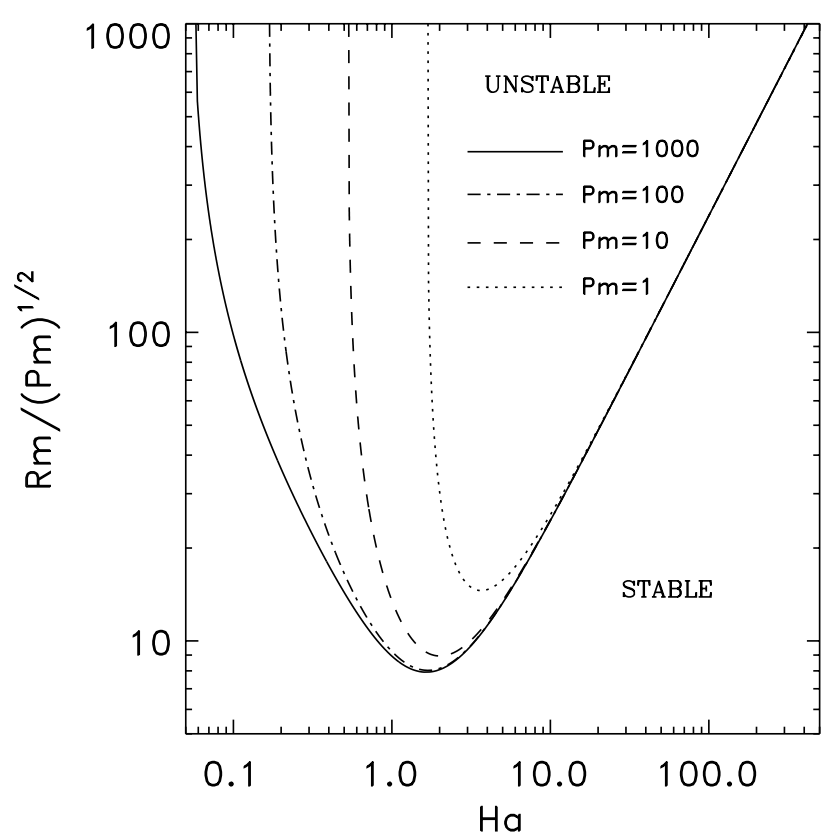

Fig. 1. The stability diagram for axisymmetric modes of quadrupole parity. The right branches and minima of the neutral stability lines remain constant for sufficiently large magnetic Prandtl numbers.

Several neutral stability lines for Pm increasing from 1 to 1000 are shown in Fig. 1. Our numerical model cannot match the huge values of Pm expected for galaxies. However, Eqs. (A.6)-(A.8) suggest (and the results of Fig. 1 confirm) that the neutral stability lines approach a universal behavior in the high Pm limit. This behavior obeys the so-called similarity rules from which the MRI characteristics for arbitrarily large Pm can be estimated.

Three similarity rules can be found from the numerical results illustrated by Fig. 1:

(i) on the right-hand branch the lines approach a constant slope for high Rm, so that one always has

$$
\frac{\mathrm{Rm}}{\mathrm{Ha} \sqrt{\mathrm{Pm}}} \equiv \frac{\Omega_{0} H}{V_{\mathrm{A}}} \simeq 2.4 \text {. }
$$

With $\Omega_{0} H / 2 \simeq c_{\mathrm{s}}$, one finds the magnetic Mach number slightly below unity;

(ii) as Pm grows, the lines approach a common minimum of

$$
\left(\frac{\mathrm{Rm}}{\sqrt{\mathrm{Pm}}}\right)_{\min } \equiv\left(\frac{\Omega_{0} H^{2}}{\sqrt{v \eta}}\right)_{\min } \simeq 8 ;
$$

(iii) the left-hand branches in Fig. 1 are close to vertical lines at large $\mathrm{Rm}$. The lines are almost equidistant in the logarithmic scale suggesting that the Lundquist number $\mathrm{S}$ is approaching a constant, i.e.

$$
\mathrm{S}=\sqrt{\mathrm{Pm}} \mathrm{Ha} \equiv \frac{V_{\mathrm{A}} H}{\eta} \simeq 1.7 .
$$

The values (10)-(12) provided by the global model do not coincide with the estimates (A.6)-(A.8) from a local treatment. The difference can be partly attributed to the different definition of the basic parameters in the local analysis (cf. Eqs. (6)
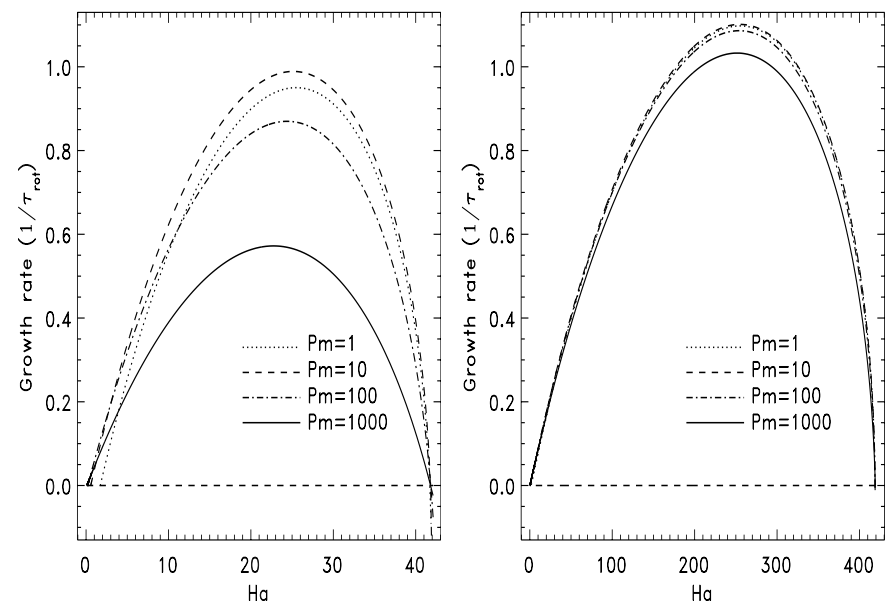

Fig. 2. Variation of the normalized growth rates $\gamma \tau_{\text {rot }}$ from the lefthand branch to the right-hand branch across the stability diagram of Fig. 1 for $\mathrm{Rm} / \sqrt{\mathrm{Pm}}=100$ (left) and $\mathrm{Rm} / \sqrt{\mathrm{Pm}}=1000$ (right).

and (A.3)). However, the scaling used for large Pm was inferred from the local analysis presented in the appendix.

Figure 2 shows how the growth rates of unstable perturbations vary along a horizontal line (constant $\mathrm{Rm} / \sqrt{\mathrm{Pm}}$ ) from the left to the right branches of the stability diagram. The growth rates are shown in units of the rotation frequency, $\tau_{\text {rot }}^{-1}=\Omega_{0} / 2 \pi$, of the disk core. It is suggested that as the Reynolds number increases the lines join together in a common dependence for different Pm. The typical growth times are of order $\tau_{\text {rot }}$.

Figure 3 illustrates the structure of the global modes. The broken line encircles the rigidly rotating core, $s_{0}=5 \mathrm{H}$. The modes are truly global with large horizontal scales. The scale, however, decreases with increasing amplitude of $B_{0}$. This behavior is accompanied by an increase of the pitch angle between the azimuthal direction and the field vector. The pitch angle is small for the minimum magnetic field. It increases to almost $90^{\circ}$ for the maximum field producing MRI. In both limits the magnetic-induced angular momentum transport vanishes.

The linear theory cannot yield the magnitude of the magnetic stress but it can compare it with the kinetic stress. The angular momentum flux by Reynolds stress is also outward but it is small compared to the Maxwell stress (cf. Brandenburg et al. 1995; Rüdiger et al. 1999). The relation reflects the magnetic nature of MRI. Also the energy of the unstable modes is dominated by its magnetic part.

Figure 4 shows the stability diagram for axisymmetric dipolar modes. Comparison with Fig. 1 reveals a preference for the quadrupole parity. The neutral stability lines of Fig. 4 lie totally inside the corresponding instability regions of Fig. 1. This means that when the system approaches instability to disturbances of dipolar parity, it is already unstable to quadrupolar perturbations. In the same sense, axisymmetric disturbances are preferred to nonaxisymmetric ones (Kitchatinov \& Mazur 1997). 


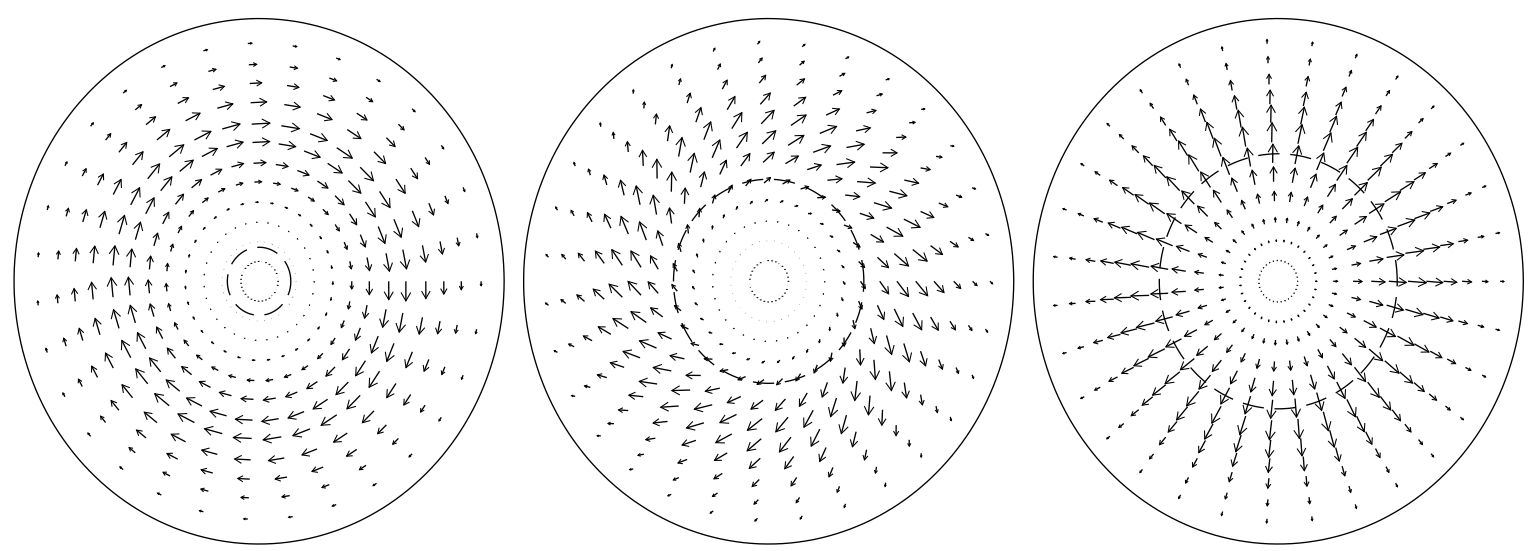

Fig. 3. Magnetic field in the midplane of the disk for the quadrupole-parity mode at $\mathrm{Rm} / \sqrt{\mathrm{Pm}}=1000$. From left to right: the marginally stable mode for the minimum magnetic field, the mode with maximum growth rate $\gamma=1.03 / \tau_{\text {rot }}$ at $\mathrm{Ha} \simeq 250$, and the marginally stable mode for maximum magnetic field. The broken circle is the radius $s_{0}=5 \mathrm{H}$ of the rigidly rotating core of the disk. The background rotation is anticlockwise. Note the increase of the pitch angle and the decrease of the horizontal scale with increasing field strength.

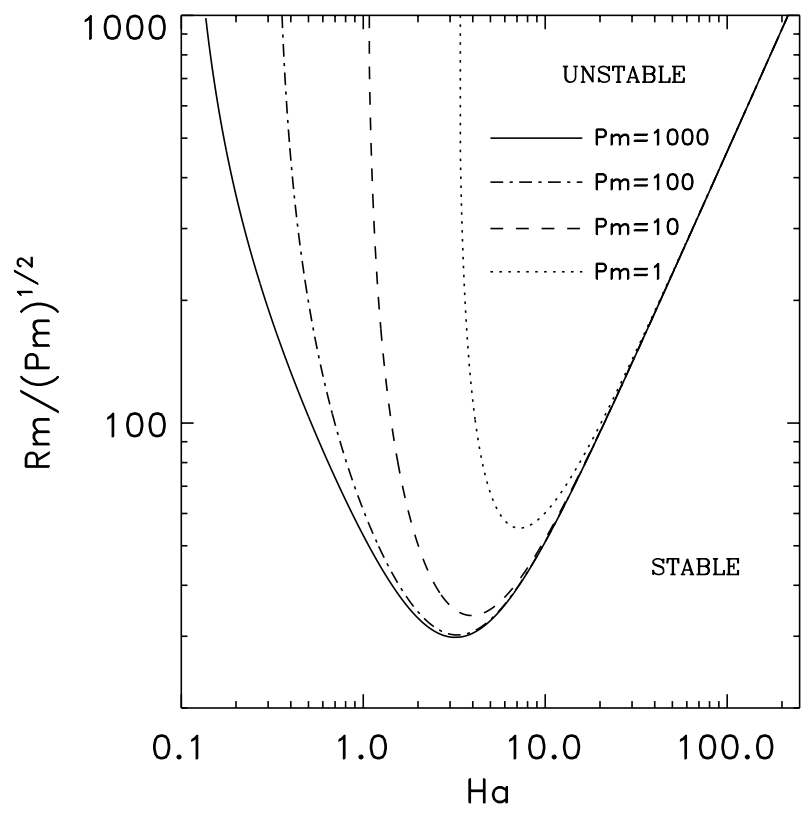

Fig. 4. The stability diagram for dipolar modes. The modes are harder to excite than the quadrupolar modes of Fig. 1.

\section{Implications for galactic dynamos}

\subsection{Basic parameters}

To probe the relevance of the MRI for galactic magnetism, the parameters controlling the instability should be estimated for galaxies.

We assume the angular velocity of the disk core as $\Omega_{0} \simeq 3 \times$ $10^{-15} \mathrm{~s}^{-1}$ which corresponds to a rotation period $\tau_{\text {rot }} \simeq 70 \mathrm{Myr}$ (Carignan et al. 1990; Sofue 1996). The half-thickness of the disk is assumed as $H \simeq 10^{21} \mathrm{~cm} \simeq 300 \mathrm{pc}$. For a plasma with not too low degree of ionization, conductivity is controlled by electron scattering on ions. The magnetic diffusivity then is,

$\eta=10^{13} T^{-3 / 2}\left(\frac{\ln \Lambda}{20}\right) \mathrm{cm}^{2} / \mathrm{s}$, where $\ln \Lambda \simeq 20$ is the Coulomb logarithm (Spitzer 1962). Adopting $T \simeq 10^{4} \mathrm{~K}$ for the characteristic temperature, we find

$\eta \simeq 10^{7} \mathrm{~cm}^{2} / \mathrm{s}$,

$\mathrm{Rm} \simeq 3 \times 10^{20}$.

Viscosity is more difficult to estimate. The expression for a fully-ionized hydrogen plasma (Spitzer 1962),

$v=6.5 \times 10^{7} \frac{T^{5 / 2}}{n_{\mathrm{p}}}\left(\frac{20}{\ln \Lambda}\right) \mathrm{cm}^{2} / \mathrm{s}$,

can be applied to HII-regions. With a proton density, $n_{\mathrm{p}} \simeq$ $1 \mathrm{~cm}^{-3}$, it yields

$v \simeq 7 \times 10^{17} \mathrm{~cm}^{2} / \mathrm{s}, \quad \quad \mathrm{Pm} \simeq 7 \times 10^{10}$.

In the warm interstellar medium, however, neutrals can provide a larger viscosity, $v_{\mathrm{n}} \sim v_{\mathrm{t}} \ell_{\mathrm{nn}}\left(v_{\mathrm{t}}\right.$ is the thermal velocity and $\ell_{\mathrm{nn}}$ is the mean free path for neutral collisions). With a neutral density, $n_{\mathrm{n}} \simeq 1 \mathrm{~cm}^{-3}$, we find

$v \simeq 10^{21} \mathrm{~cm}^{2} / \mathrm{s}, \quad \quad \mathrm{Pm} \simeq 10^{14}$.

Fortunately, accuracy in estimating the viscosity is not important. The viscosity value is needed only to ensure that the minimum ratio Eq. (11) for instability is exceeded. Combining Eq. (14) with Eqs. (16) or (17), we confirm that the magnetic Reynolds number exceeds the critical value given by Eq. (11) by many orders of magnitude. This suggests that the range of magnetic field strengths producing MRI should be very broad.

The maximum magnetic field for instability results from Eq. (10),

$B_{\max } \simeq 6 \mu \mathrm{G}$

(cf. Sellwood \& Balbus 1999), which is surprisingly close to the observed strengths of galactic fields.

The minimum field strength for MRI can be estimated from Eq. (12) as the extremely small value

$B_{\min } \simeq 10^{-25} \mathrm{G}$. 


\subsection{Seed field amplification}

Magnetic field amplification by a hydromagnetic dynamo needs a seed field to start from. The origin of such a seed field for galaxies is very uncertain. Three possibilities can currently be found in the literature. First, the battery effect of Biermann can produce the seed field. The corresponding amplitude was estimated by Davis \& Widrow (2000) and Gnedin et al. (2000) to be about $10^{-20} \mathrm{G}$ in collapsing protogalaxies. Second, collisional friction between ions and neutrals on ionization fronts may produce fields of order $10^{-16} \mathrm{G}$ (Birk et al. 2002). Third, the cosmological magnetic field might be as strong as $10^{-10} \mathrm{G}$ (Ratra 1992). All the quoted field strengths can be further amplified by a factor $\sim 10^{2} \ldots 10^{3}$ during the collapse phase of the protogalaxy.

Whatever the origin of the seed field, the newly formed galaxies are well inside the instability region between the limits given in Eqs. (18) and (19). The region should be entered somehow, hence the instability should start even before the galaxies are formed. MRI in young galaxies is operating in the far supercritical region, $B \gg B_{\min }$. Growth times of order $\tau_{\text {rot }}$ are expected for this case. The maximum growth rates initially belong to small spatial scales, $\ell \ll H$, with $\Omega_{0} \ell \simeq V_{\mathrm{A}} \gg \eta / \ell$ (Fricke 1969; Balbus \& Hawley 1991). The scale range certainly exists for the very large values given by Eq. (14) $2_{2}$. MRI at its nonlinear stage is known to be able to amplify the magnetic field producing the instability. As the unstable perturbations grow, the increase of their magnetic field should shift the maximum growth rates to larger and larger scales until the global perturbations start dominating.

The turbulent amplification of magnetic fields was studied by Beck et al. (1994). They did not specify the origin of turbulence in favor of quoting observational evidence for the turbulence. We argue here that the MHD turbulence can be produced by MRI.

If we assume that the nonlinear amplification of magnetic fields proceeds with about the same rate as the linear growth, the seed field can be amplified by a factor of $f_{\text {amp }}\left(\tau_{\text {gal }}\right) \simeq \exp \left(\tau_{\text {gal }} / \tau_{\text {rot }}\right)$ for a galactic age $\tau_{\text {gal }}$. With the value $\Omega_{0} \simeq 3 \times 10^{-15} \mathrm{~s}^{-1}$ it takes less than $2 \mathrm{Gyr}$ to produce a $1 \mu \mathrm{G}$ field from even the weakest seed field expected from the Biermann battery effect. This may explain the regular magnetic fields of $\mu \mathrm{G}$ strength for galaxies with high redshift $z \simeq 2$ $\left(\tau_{\text {gal }} \sim 1 \ldots 3 \mathrm{Gyr}\right)$. Restrictions on the magnitude of the seed field, however, would emerge if the microgauss fields were already present in very young galaxies as found at $z \simeq 3.4$ by White et al. (1993).

\subsection{Field geometry}

We saw in Sect. 3 that MRI preferentially produces global perturbations of quadrupolar parity although the background field for the instability is dipolar. Therefore, MRI provides a seed field of "correct" parity for global dynamos. It may also be mentioned that the pitch angles (the angles between the azimuthal direction and the field vector) in $\alpha \Omega$-dynamo models are often too small. The small angles result from an efficient winding of the magnetic field by the nonuniform rotation.
Figure 3 shows that the pitch angles for the most rapidly growing global modes of MRI are not small.

It is characteristic for linear global MRI models that the symmetries of flow and field always differ. If the magnetic field is symmetric with respect to the midplane then the flow is antisymmetric and vice versa. Hence, if the global MRI is really present in galaxies, then the associated flow field is antisymmetric with respect to the midplane. Then the effective rotation rate would differ between hemispheres. Nonlinear calculations must show whether in the final, saturated phase these characteristic mixed-mode properties of MRI-induced magnetism survive. It might be important in this connection that in an evolving galaxy with $\mathrm{SN}$-explosions a second driver of interstellar turbulence occurs which may finally dominate the magneticfield production.

Acknowledgements. We wish to thank our referee Katia Ferrière for constructive comments. L.L.K. is grateful to A.I.P. for its hospitality and visitor support. This work was supported by INTAS under grant No. 2001-0550 and by the Russian Foundation for Basic Research (Project 02-02-16044).

\section{Appendix A: Local analysis of marginal stability}

The guidance for appropriate scaling of our numerical results came from an elementary local analysis. The local treatment of MRI provides expectations and better understanding for global simulations.

We use a Cartesian coordinate system corotating with local angular velocity, $\Omega$, and axes $x, y$ and $z$ pointing in the radial, azimuthal and vertical directions respectively. The local approximation concerns perturbations with scales small compared to the global scale. Then, the rotation law can be approximated by the shear flow $\boldsymbol{U}_{0}=-\hat{\boldsymbol{e}}_{y} \Omega q x$ where $q$ is the (constant) local shear. The linearized MHD equations in the local approximation read

$$
\begin{aligned}
\frac{\partial \boldsymbol{B}^{\prime}}{\partial t} & -B_{0} \frac{\partial \boldsymbol{u}^{\prime}}{\partial z}-x q \Omega \frac{\partial \boldsymbol{B}^{\prime}}{\partial y}+q \Omega B_{x}^{\prime} \hat{\boldsymbol{e}}_{y}-\eta \Delta \boldsymbol{B}^{\prime}=0 \\
\frac{\partial \boldsymbol{u}^{\prime}}{\partial t} & +2 \Omega \hat{\boldsymbol{e}}_{z} \times \boldsymbol{u}^{\prime}-x q \Omega \frac{\partial \boldsymbol{u}^{\prime}}{\partial y}-q \Omega u_{x}^{\prime} \hat{\boldsymbol{e}}_{y} \\
& -\frac{B_{0}}{\mu_{0} \rho} \frac{\partial \boldsymbol{B}^{\prime}}{\partial z}+\frac{1}{\rho} \nabla P^{\prime}-v \Delta \boldsymbol{u}^{\prime}=0
\end{aligned}
$$

(Balbus \& Hawley 1991; Brandenburg et al. 1995) where $P^{\prime}$ is the pressure fluctuation including the magnetic term, $B_{0}$ is the amplitude of the background axial magnetic field, $\boldsymbol{B}_{0}=B_{0} \boldsymbol{e}_{z}$.

Considering plane waves with $\boldsymbol{B}^{\prime}, \boldsymbol{u}^{\prime}, P^{\prime} \sim \exp (\gamma t+\mathrm{i} k z)$ leads to the dispersion equation,

$$
\begin{aligned}
& \left(\gamma+\eta k^{2}\right)^{2}\left(\left(\gamma+v k^{2}\right)^{2}+2(2-q) \Omega^{2}\right) \\
& \quad+\omega_{\mathrm{A}}^{2}\left(\omega_{\mathrm{A}}^{2}-2 q \Omega^{2}+2\left(\gamma+v k^{2}\right)\left(\gamma+\eta k^{2}\right)\right)=0
\end{aligned}
$$

with the Alfvén frequency $\omega_{\mathrm{A}}=k B_{0} / \sqrt{\mu_{0} \rho}$.

We need the boundary separating the regions of stability and instability in parameter space. The magnetic Reynolds number and Hartmann number have to be redefined for the local treatment as

$$
\mathrm{Rm}=\frac{\Omega}{\eta k^{2}}, \quad \mathrm{Ha}=\frac{\omega_{\mathrm{A}}}{k^{2} \sqrt{\eta v}} .
$$


To find the marginal stability equation we put $\gamma=0$ in Eq. (A.2). After some algebra this yields

$\mathrm{Rm}^{2}=\frac{\mathrm{Pm}^{2}\left(1+\mathrm{Ha}^{2}\right)^{2}}{2\left(q \mathrm{Pm} \mathrm{Ha}^{2}-2+q\right)}$.

With a fixed value of Pm, this equation leads to neutral stability lines similar to those of Fig. 1. For a given sufficiently large $\mathrm{Rm}$, the instability exists in the region between some minimum and maximum values of $\mathrm{Ha}$. We call the lines defining the minimum and maximum Ha the left and the right branches of the marginal stability line.

Then the minimum Reynolds number for instability is

$\mathrm{Rm}_{\text {min }}^{2}=\operatorname{Pm} \frac{2}{q}\left(1+\frac{2-q}{q \operatorname{Pm}}\right)$

at

$\mathrm{Ha}^{2}=1+\frac{2(2-q)}{q \mathrm{Pm}}$.

For large $\mathrm{Pm}$, the ratio of $\mathrm{Rm} / \sqrt{\mathrm{Pm}}$ at minimum approaches a constant and so does the corresponding Ha. This behavior at large Pm agrees with the numerical results of Sect. 3. Actually, the scaling used in that section was motivated by the local analysis.

The right branch of the neutral stability lines at high enough $\mathrm{Rm}$ can be found by considering Eq. (A.4) for large Ha,

$\mathrm{Rm}^{2}=\frac{\mathrm{Pm}}{2 q} \mathrm{Ha}^{2}$

Again the ratio of $\mathrm{Rm} / \sqrt{\mathrm{Pm}}$ is an appropriate scaling for the slope of the right branch.

The left branch is controlled by the diffusivities. Its expression for large Rm can be obtained by by setting the denominator in Eq. (A.4) to zero.

$B_{\min }^{2}=\frac{2-q}{q} \mu_{0} \rho \eta^{2} k^{2}$.

The smallest magnetic field producing MRI belongs to the smallest possible wave number so that also the left branch is controlled by global disturbances. Equation (A.8) shows that the minimum Lundquist number is constant on the left branch for sufficiently high Rm.

\section{References}

Athreya, R. M., Kapahi, V. K., McCarthy, P. J., \& van Breugel, W. 1998, A\&A, 329, 809

Balbus, S. F., \& Hawley, J. F. 1991, ApJ, 376, 214

Beck, R., Poezd, A. D., Shukurov, A., \& Sokoloff, D. D. 1994, A\&A, 289, 94

Birk, G. T., Weichen, H., \& Lesch, H. 2002, A\&A, 393, 685

Brandenburg, A., Nordlund, Å., Stein, R. F., \& Torkelsson, U. 1995, ApJ, 446, 741

Carignan, C., Charbonneau, P., Boulanger, F., \& Viallefond, F. 1990, A\&A, 234, 43

Carilli, C. L., \& Taylor, G. B. 2002, ARA\&A, 40, 319

Cattaneo, F. 1999, ApJ, 515, L39

Davis, G., \& Widrow, L. M. 2000, ApJ, 540, 755

Ferrière, K. 1992, ApJ, 391, 188

Fricke, K. 1969, A\&A, 1, 388

Gnedin, N. Y., Ferrara, A., \& Zweibel, E. G. 2000, ApJ, 539, 505

Kaisig, M., Rüdiger, G., \& Yorke, H. W. 1993, A\&A, 274, 757

Kitchatinov, L. L., \& Mazur, M. V. 1997, A\&A, 324, 821

Krause, F., \& Beck, R. 1998, A\&A, 335, 789

Kulsrud, R. M., \& Anderson, S. W. 1992, ApJ, 396, 606

Lesch, H., \& Hanasz, M. 2003, A\&A, 401, 809

Ratra, B. 1992, ApJ, 391, L1

Rüdiger, G., Primavera, L., Arlt, R., \& Elstner, D. 1999, MNRAS, 306, 931

Rüdiger, G., \& Hollerbach, R. 2004, The Magnetic Universe (Weinheim: Wiley)

Schekochihin, A. A., Cowley, S. C., Maron, J. L., \& McWilliams, J. C. 2004, Phys. Rev. Lett., 92, 054502

Sellwood, J. A., \& Balbus, S. A. 1999, ApJ, 511, 660

Sofue, Y. 1996, ApJ, 458, 120

Spitzer, L. 1962, Physics of fully ionized gases (New York: Wiley)

Stix, M. 1975, A\&A, 42, 85

Velikhov, E. P. 1959, Sov. Phys. JETP, 9, 995

White, R. L., Kinney, A. L., \& Becker, R. H. 1993, ApJ, 407, 556

Wolfe, A. M. 1988, Damped Ly-alpha absorption systems, in QSO absorption lines: Probing the Universe, ed. C. Blades, D. A. Turnshek, \& C. A. Norman (Cambridge: Cambridge Univ. Press), 297 